\title{
Suitability of Artificial Neural Networks for Designing LoC Circuits
}

\author{
David Moreno, Sandra Gómez, and Juan Castellanos \\ Natural Computing Group, Technical University of Madrid, \\ Campus de Montegancedo, 28660 Boadilla del Monte (Madrid) Spain \\ d.mnavas@alumnos.upm.es, sgomez@eui.upm.es, jcastellanos@fi.upm.es
}

\begin{abstract}
The simulation of complex LoC (Lab-on-a-Chip) devices is a process that requires solving computationally expensive partial differential equations. An interesting alternative uses artificial neural networks for creating computationally feasible models based on MOR techniques. This paper proposes an approach that uses artificial neural networks for designing LoC components considering the artificial neural network topology as an isomorphism of the LoC device topology. The parameters of the trained neural networks are based on equations for modeling microfluidic circuits, analogous to electronic circuits. The neural networks have been trained to behave like AND, OR, Inverter gates. The parameters of the trained neural networks represent the features of LoC devices that behave as the aforementioned gates. This would mean that LoC devices universally compute.
\end{abstract}

Keywords: LoC, Lab-on-a-Chip, MOR, Microfluidic devices, Nanofluidic devices, Artificial neural networks.

\section{Introduction}

Lab-on-a-Chip (LoC) is a field of research and technological development with the goal of constructing highly integrated compact devices for integrating multiple laboratory functions on a single chip with a minimized size. The integration is carried out on monolithic platforms which permit the integration of micro(nano)fluidic functionalities and components necessary to accomplish one or more biochemical or chemical processes. Microfluidic lab-on-a-chip (LoC) systems have been studied for more than a decade and have many applications in biology, medicine, and chemistry $[7,8]$. LoC devices perform chemical analysis involving sample preparation, mixing, reaction, injection, separation analysis and detection [1]. The most highly integrated Lab-on-a-Chip devices include all processes and devices on a single chip or card so that the introduction of an unprocessed sample leads to the output of an analytical result - an "answer" from that same chip [4].

Simulating these types of devices and their components requires a great number of parameters and complex partial differential equations. An efficient way of simulating LoC devices involves functional decomposition into a series of interconnected blocks which work to create models for use in the decomposition when 
first principle models are not possible. These blocks are the mixer, the injector, and the separator. For the mixer and separator, band shape assumptions are used with analytical techniques for simplifying the partial differential equations into several ordinary differential equations. For the injector, the resulting modeled outputs are described by a finite number of performance functions; hence numerical techniques are used to describe these functions in a finite domain (see [4]).

The structure of this paper is as follows. Section 2 is the background where we present the theoretical basis that supports the approach proposed by other researchers [4] who use artificial neural networks for simulating the behavior of components of a set of microfluidic devices. Section 3 presents our proposal for designing LoC devices through artificial neural networks which map the topology of the LoC. Section 4 presents conclusions and future work.

\section{Artificial Neural Networks for Simulating LoC Components}

Simulation of complex LoC devices is a very expensive process from the point of view of computational resources. In general, numerical solutions for partial differential equations are necessary to achieve an appropriate simulation. Processing these solutions is costly. As the design of LoC systems requires many repeated simulations, iterative design using numerical simulation is computationally infeasible [1]. The proposal [2] simplifies this process applying MOR (Model Order Reduction) for splitting the spatial dependency of device behavior, extracting the most typical characteristics of the governing equations and, hence, reduces the complexity of the problem. In particular, applying MOR is undertaken to reduce the number of parameters in the simulation. The methodology proposed in [1] uses the results of MOR applied to specific LoC components as input for training an artificial neural network. This trained neural network simulates the behavior and performance of the specified LoC components. Finally, the result obtained is a model for designing LoC components generated through the neural architecture. This approach is tested in [1] modeling an injector component because it defines the shape and quantity of analytes that will be used for separation and analysis. The injector components modeled were cross, double-tee and gated-cross. Modeling an injector is a very difficult task. The approach [1] tries to simplify the mathematical model maintaining accuracy with respect to physical features. The steps for modeling the injector described in [1] are

1. The $\pi$ - Buckingham theorem is used for reducing the physical parameter space. The reduced parameters are dimensionless.

2. The process is carried out in the dimensionless parameter space to obtain the minimal number of numerical simulations and so reduce cost.

3. An artificial neural network is constructed to analytically describe the parameter space.

(a) The selected network topology is feed-forward back-propagation. 
(b) The network learns the functional mapping without knowing the underlying physical basis.

4. The trained network is converted into an explicit algebraic function appropriate for use in any software environment for executing a simulation or synthesis process.

The results obtained in this research indicate that the injector model obtained by neural networks is very close to the result obtained by numerical simulations. This result is fascinating because, as described above, numerical simulations are not only expensive to carry out but time-consuming. This approach demonstrates that artificial neural networks can obtain models very close to the results obtained in numerical simulations at lower resources cost.

\section{Artificial Neural Networks for Designing LoC Devices}

The main idea involves considering the topology of an artificial neural network as an isomorphism between this network and a microfluidic device (LoC). This isomorphism permits the network to be trained in a specific function and to transform elements such as nodes, edges, weights and activation functions into elements and features of a microfluidic device. This approach is based on the analogy between electronic circuits and microfluidic circuits shown in the next equation (for more details, see [3])

$$
\Delta P=L_{f} \frac{d Q}{d t}+R_{f} Q+\frac{1}{C_{f}} \int Q d t
$$

Where $P$ is pressure, $t$ is time, $Q$ is the rate of volumetric flow, $L$ is inductance (due to the inertia of the fluid), $R$ is resistance (due to the transversal forces of the channel walls), $C$ is capacity (due to the compressibility of the fluid). In most of the microfluidic circuits, the third member of equation 1 can be omitted.

For a channel with transversal fixed section and circular shape, fluidic resistance is expressed by

$$
R_{f}=\frac{8 \mu l}{\pi r_{c}^{4}}
$$

Where $\mu$ is the viscosity dynamic of the fluid, $l$ is the length of the channel and $r_{c}$ is the radius of the channel.

Fluidic inductance is defined by

$$
L_{f}=\frac{\rho l}{A_{c}}
$$

Where $\rho$ is the density of the fluid, $l$ is the length o the channel and and $A_{c}$ is the transversal area of the channel.

Now, $Q$ can be expressed in the following way

$$
Q=G_{f} \Delta P
$$


Where $G_{f}$ is the fluidic conductance, $Q$ can be expressed as the sum of input rates of volumetric flow

$$
Q=\sum_{i} Q_{i}
$$

Based on the equations above, we can model each component of the neural network with the following features:

- The whole of the nodes are LoC chambers where reactions and processes take place.

- The input nodes receive rates of volumetric flow $Q_{i}$ as inputs.

- The weights of the edges are $R_{f i j}$. This paper considers the rate of volumetric flow $Q$ as constant, so $L_{f}$ is not modeled.

- The output nodes return $\Delta P_{j}=\sum_{i} Q_{i} R_{f i j}$ as output.

Artificial neural networks have been trained to behave like AND, OR and inverter gates. The possible values and the equivalences between digital inputs and flows are

- 0 is equal to any value lower than a flow unit

- 1 is equal to any value equal or greater than a flow unit.

\subsection{AND Gate}

The truth table of the AND gate is shown in Table 1

Table 1. Truth Table of the AND Gate

$$
\begin{array}{|l|l|l|}
\hline x_{1} & x_{2} & y \\
\hline \hline 0 & 0 & 0 \\
\hline 0 & 1 & 0 \\
\hline 1 & 0 & 0 \\
\hline 1 & 1 & 1 \\
\hline
\end{array}
$$

A simple artificial neural network that implements this gate has two input nodes and one output node, with two edges, each from input node to output node. The weights of the edges are equal to 0.5 and the threshold for the activation function is 1 . With these values, the equation for fluidic resistance is

$$
R_{f}=\frac{8 \mu l}{\pi r_{c}^{4}}=0,5
$$

Variables can take different values. This represents the degrees of freedom provided in terms of the possibilities that the lab-on-chip design permits. The dynamic viscosity of fluids can be determined by fixing the radius channel and its length with known resistance 


$$
\mu=\frac{0,5 \pi r_{c}^{4}}{8 l}
$$

Similarly, it is possible to determine the radius channel by fixing the dynamic viscosity, channel length and resistance

$$
r_{c}=\sqrt[4]{\frac{\mu 8 l}{0,5 \pi}}
$$

Finally, it is possible to determine the length of the channel by fixing its radius, the dynamic viscosity and resistance

$$
l=\frac{0,5 \pi r_{c}^{4}}{8 \mu}
$$

The value for the output node is

$$
\Delta P=0,5\left(Q_{1}+Q_{2}\right)
$$

If the pressure applied is greater than or equal to the unit of pressure, the flow through the node is the desired one.

\subsection{OR Gate}

The truth table of the OR gate is shown in Table 2

Table 2. Truth table of the OR gate

$$
\begin{array}{|l|l|l|}
\hline x_{1} & x_{2} & y \\
\hline \hline 0 & 0 & 0 \\
\hline 0 & 1 & 1 \\
\hline 1 & 0 & 1 \\
\hline 1 & 1 & 1 \\
\hline
\end{array}
$$

A simple artificial neural network that implements this gate has two input nodes and one output node, with two edges, each from input node to output node. The weights of the edges are equal to the value 1 and the threshold for the activation function is 1 .

With these values, the next equation for the fluidic resistance is

$$
R_{f}=\frac{8 \mu l}{\pi r_{c}^{4}}=1
$$

Variables can take different values. This represents the degrees of freedom provided in terms of the possibilities that the lab-on-chip design permits. The dynamic viscosity of fluids can be determined by fixing the radius channel and its length with known resistance 


$$
\mu=\frac{\pi r_{c}^{4}}{8 l}
$$

Similarly, it is possible to determine the radius channel by fixing the dynamic viscosity, channel length and resistance

$$
r_{c}=\sqrt[4]{\frac{\mu 8 l}{\pi}}
$$

Finally, it is possible to determine the length of the channel by fixing its radius, the dynamic viscosity and resistance

$$
l=\frac{\pi r_{c}^{4}}{8 \mu}
$$

The value for the output node is

$$
\Delta P=Q_{1}+Q_{2}
$$

If the pressure applied is greater than or equal to the unit of pressure, flow through the node is the desired flow.

\subsection{Inverter}

Inspired by "NOT A AND B" gate in [6], this is an inverter gate adapted to the features of LoC devices. We consider it impossible to have negative resistance, but the flows can interfere with one another. The base of the inverter is an artificial neural network that implements an XOR gate with an input always equal to 1. Figure 1 (obtained with JavaNNS [5]) shows the network used to create our inverter, where the thresholds of the activation functions are below those of the hidden nodes and $y$.

Let $x_{1}$ be the input which always has the value 1 , and let $x_{2}$ be the input to be inverted. Table 3 shows the truth table of the XOR gate where the rows of interest are in italics, i.e. those whose $\mathrm{x} 1$ input is equal to 1 . Fluidic resistance does not have negative values, so we can consider the negative weights as channels from input flow to channels whose weights in the neural network are positive; thus the channels, with negative weight-resitance, will oppose the positive flow. In the Figure 1, a "negative" channel goes to the channel represented in the neural network through the edge from the input $x 1$ to the node in the hidden layer.

The values for $R_{f}$ to be considered for the each channel are (with absolute values)

$$
\begin{gathered}
R_{f x_{1} \text { hidden }}=\frac{8 \mu l}{\pi r_{c}^{4}}=6.508 \\
R_{f x_{1} y}=\frac{8 \mu l}{\pi r_{c}^{4}}=4.661
\end{gathered}
$$




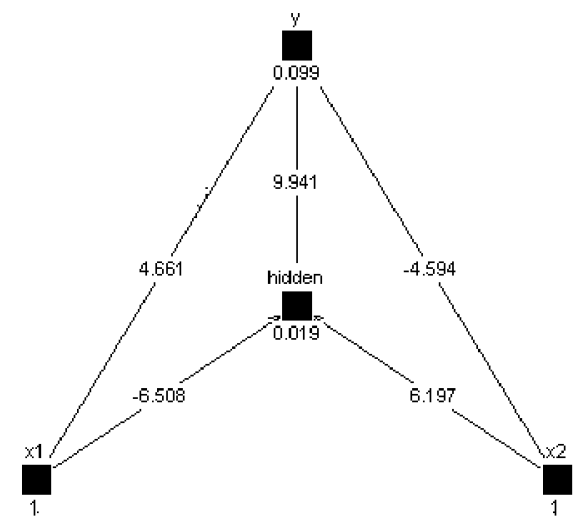

Fig. 1. XOR Network

Table 3. Truth Table of the XOR gate

$$
\begin{aligned}
& \begin{array}{|l|l|l|}
\hline x_{1} & x_{2} & y \\
\hline \hline 0 & 0 & 0 \\
\hline 0 & 1 & 1 \\
\hline 1 & 0 & 1 \\
\hline 1 & 1 & 0 \\
\hline
\end{array} \\
& R_{f x_{2} \text { hidden }}=\frac{8 \mu l}{\pi r_{c}^{4}}=6.197 \\
& R_{f x_{2} y}=\frac{8 \mu l}{\pi r_{c}^{4}}=4.594 \\
& R_{\text {hidden } y}=\frac{8 \mu l}{\pi r_{c}^{4}}=9.941
\end{aligned}
$$

The values for the $\Delta P$, both hidden node and output node are

$$
\begin{gathered}
\Delta P_{\text {hidden }}=R_{f x_{1} \text { hidden }} Q_{1}+R_{f x_{2} \text { hidden }} Q_{2} \\
\Delta P_{y}=R_{f x_{1} y} Q_{1}+\Delta P_{h i d d e n}+R_{f x_{2} y} Q_{2}
\end{gathered}
$$

\section{Conclusions and Future Work}

This paper has presented, in section 2, other studies demonstrating the viability of neural networks for simulating lab-on-a-chip (LoC) components. The contribution of this paper is to demonstrate the suitability of mapping artificial neural network topology into a lab-on-a-chip (LoC) using the neural network as a design 
tool. AND, OR and inverter gates have been used to prove the potential of this approach. It is important to emphasize that these three gates are necessary for a device, abstract or real, to compute universally. In this sense, it is possible to affirm this approach makes universal computation in a lab-on-a-chip (LoC) conceivable. In general, this idea opens possibilities for designing any type of circuit to be used to represent the functionalities performed in a lab-on-a-chip (LoC). Our approach permits the creation of more complex operations over samples e.g. "if element $x_{1}$ is present and element $x_{2}$ is not but element $x_{3}$ is, the microfluidic device must do a specific task" in a straightforward way. The circuits designed using this methodology should be tested by means of lab-ona-chip (LoC) simulation tools. Comparisons between theoretical and simulated results could support this new methodology. Future research can be focused on taking into account the first element of equation 1, i.e. the influence of inductance with respect to the variation of volumetric flow over time in pressure. Neural networks able to solve differential equations could be used for this purpose.

\section{References}

1. Magargle, R., Hoburg, J.F., Mukherjee, T.: Microfluidic injector models based on artificial neural networks. In: Chakrabarty, K., Zeng, J. (eds.) Design Automation Methods and Tools for Microfluidics-Based Biochips, pp. 215-233. Springer, Heidelberg (2006)

2. Wang, Y., Bedekar, A.S., Krishnamoorthy, S., Sundaram, S., Singhal, A.K.: Model Order Reduction (MOR). In: Li, D. (ed.) Encyclopedia of Microfluidics and Nanofluidics, pp. 1382-1391. Springer, Heidelberg (2008)

3. Dutta, P., Horiuchi, K., Jubery, T.Z.: Microfluidic Circuits. In: Li, D. (ed.) Encyclopedia of Microfluidics and Nanofluidics, pp. 1151-1158. Springer, Heidelberg (2008)

4. Garcia-Cordero, J.L., Ricco, A.J.: Lab-on-a-Chip (General Philosophy). In: Li, D. (ed.) Encyclopedia of Microfluidics and Nanofluidics, pp. 962-969. Springer, Heidelberg (2008)

5. JavaNNS (Java Neural Network Simulator), http://ww.ra.cs.uni-tuebingen.de/software/JavanNS

6. Prakash, M., Gershenfeld, N.: Microfluidic Bubble Logic. Science 315(5813), 832-835 (2007)

7. Reyes, D.R., Iossifidis, D., Auroux, P.A., Manz, A.: Micro Total Analysis Systems. 1. Introduction, Theory and Technology. Anal. Chem. 74(12), 2623-2636 (2002)

8. Auroux, P.-A., Iossifidis, D., Reyes, D.R., Manz, A.: Micro Total Analysis Systems. 2. Analytical Standard Operations and Applications. Anal. Chem. 74(12), $2637-$ 2652 (2002) 